Revue d'histoire de l'Amérique française

RAS REVUE D.HISTOIRE DE L'AMÉRIQUE FRANÇAISE

\title{
Note sur la chapellerie au Canada sous le régime français
}

\section{Lionel Groulx}

Volume 3, numéro 3, décembre 1949

URI : https://id.erudit.org/iderudit/801578ar

DOI : https://doi.org/10.7202/801578ar

Aller au sommaire du numéro

Éditeur(s)

Institut d'histoire de l'Amérique française

ISSN

0035-2357 (imprimé)

1492-1383 (numérique)

Découvrir la revue

Citer cet article

Groulx, L. (1949). Note sur la chapellerie au Canada sous le régime français. Revue d'histoire de l'Amérique française, 3(3), 383-401.

https://doi.org/10.7202/801578ar d'utilisation que vous pouvez consulter en ligne.

https://apropos.erudit.org/fr/usagers/politique-dutilisation/ 


\section{NOTE SUR LA CHAPELLERIE AU CANADA SOUS LE RÉGIME FRANÇAIS}

On connaît l'histoire de cette chapellerie. En 1736 deux chapeliers opéraient: l'un à Québec, Barthélémy Coton; l'autre, dans l'un des faubourgs de Montréal, Joseph Hupé, dit la Groy. Un troisième, de Montréal même, Jean-Baptiste Chauffour, ancien chapelier de Paris, se préparait à mettre en train une boutique. Coton et Hupé fabriquaient des chapeaux de castor demi-foulés. ${ }^{1}$ Auraient-ils conçu la dessein de développer leur petite industrie, - Coton n'avait que 400 livres de gages - et d'aller concurrencer sur leur marché, les chapeliers de Paris? Il semble bien qu'il en fut ainsi, au moins d'une certaine façon. Avis, en tout cas, fut donné en 1734 à la Compagnie des Indes qu'un plan venait d'être formé au Canada "pour fabriquer des Chapeaux a demi-foulés, et les Faire passer en France, ou ils seroient perfectionnés". ${ }^{2}$ Mal en prit aux audacieux. Tous trois, en septembre 1736, recevaient la visite du lieutenant-général civil et criminel de leur ville, accompagné d'un greffier et d'estimateurs. Ces officiers de la juridiction royale avaient ordre du gouverneur Beauharnois et de l'intendant Hocquart de détruire les établis à fouler les chapeaux, et après estimation du tout, de transporter les ustensiles aux magasins du roi. Beauharnois et Hocquart agissaient au surplus d'après l'ordre du roi. Chauffour, dont l'établissement restait à l'état de projet, en fut quitte pour un procès-verbal. Quant aux autres - détail cocasse: Coton était receveur de la Compagnie des Indes, à Québec, employé par conséquent du monopole du castor -, Coton, de même que Hupé dit la Groy, et celui-ci en dépit de sa dévotieuse enseigne qu'il faisait flotter au vent: $A$ u chapeau royal, durent assister à la démolition de leur petite industrie: établis, fourneaux, chaudières à fouler et à teindre. ${ }^{3}$

1. Fouler un chapeau, c'est le presser dans un bain d'eau, de tartre blanc, etc., pour que l'étoffe se feutre. "On feutre en foulant" (Dictionnaire Bescherelle).

2. Le ministre à M. de Fulvy, 22 déc. 1735, AC, B 62: 113-16.

3. BRH (Bulletin des recherches historiques), 28 (1922): 193-200. - AC, C 11A, 65: 10-16. 
Que s'était-il passé? Hupé dit la Groy, comme il le dépose luimême, fabriquait depuis cinq ans, en toute bonne foi. ${ }^{4}$ L'industrie de la chapellerie existait au Canada, à tout le moins, à l'époque de Talon et suscitée par l'intendant lui-même. En dépit de sa politique protectionniste et bien que fondateur de la grande industrie de France, Colbert tenait qu'il fallait mettre la colonie "en estat de se passer de toute sorte de secours". ${ }^{5}$ La Relation des Jésuites de 1666-1668, après avoir observé qu'un pays "ne peut pas se former entièrement sans l'assistance des manufactures", nous apprend que "déjà celle des soulliers et des chapeaux [sont] commencées..." 6 Talon écrit d'ailleurs au roi qu'il a "des productions du Canada de quoy [se] vestir du pied a la teste". ${ }^{7}$ Des chapeliers, il en existait au Canada avant et après Talon. D'après Beauharnois et Hocquart, on pouvait faire remonter à plus de cinquante ans la fabrication des chapeaux au Canada. ${ }^{8}$ En effet, un nommé Quénet, s'adonnait à ce métier vers 1687 à Québec, puis à Lachine. Avant lui, Honoré Langlois, dit Lachapelle, aurait aussi opéré a Montréal après $1651 .^{\circ}$

Outre Chauffour et Hupé dit la Groy, rien qu'à Montréal, E.-Z. Massicotte relève la présence d'au moins sept autres de ces fabricants. ${ }^{10}$ A l'aide des documents officiels, M. Joseph-Noël Fauteux en recense trois à Québec; et il semble qu'à Montréal, quelques Anglais, pour dissimuler leur contrebande du castor, se soient adonnés à la chapellerie. ${ }^{11} \mathrm{Il}$ ne faut pas oublier qu'en 1700 les colons avaient ressaisj, a leur profit, le commerce des fourrures et qu'un arrêt du Conseil d'Etat du 9 février de cette année-là, leur cédait le privilège de vendre

4. BRH, 28 (1922): 198.

5. RAPQ (Rapport de l'Archiviste de la province de Quebec), (1930-1931): 144.

6. JR (Jesuits Relations and Allied Documents), Ed. R.G. Thwaites (73 vol., Cleveland, 1896-1901), LI: 179.

7. RAPQ (1930-1931): 160 .

8. AC, B 62: 113-16.

9. Paul-Emile Renaud, Les Origines économiques du Canada (Mamers, 1928), 383-84. Cet ouvrage de M. Renaud reste sûrement l'étude la plus fouillée qu'on ait encore écrite sur la vie économique du Canada au temps du régime français. Il est regrettable que, faute d'avoir été revues, sans doute, ou faute d'épreuves bien corrigées, trop de références soient inexactes. Ainsi, pour cette affaire des chapeliers, le renvoi au Bulletin des recherches historiques (note 3, p. 348) ne doit pas être libellé: BRH, 27: 193, 195, 196, mais exactement: 193-200. On nous renvoie encore d BRH, 28: 373. Or rien ne s'y trouve sur la chapellerie à cette page.

10. BRH, 28 (1922): 193-94.

11. Joseph-Noël Fauteux, Essai sur l'Industrie au Canada sous le régime français (2 vol., Québec, 1927), 2: 483-85. 
leurs castors, en peau, en poil ou en chapeaux en Hollande, en Suède, au Danemark, dans les villes Hanséatiques, dans les ports de la Baltique, en Moscovie. ${ }^{12}$ Quoj de plus propre à susciter, dans la colonie, l'industrie de la chapellerie?

Encore une fois que s'était-il passé en 1736 ? Mis au fait de l'entreprise des chapeliers canadiens, le roi - c'est une façon de parler agit de la façon la plus cassante. Sa Majesté passe outre à toute représentation; elle n'a de cesse que la pauvre petite industrie ne soit littéralement foudroyée, anéantie. ${ }^{13} \mathrm{En}$ cet incident de l'histoire économique du Canada sous l'ancien régime, incident resté unique par son excessive rigueur, faut-il voir une juste expression de la politique métropolitaine à l'égard de l'industrie coloniale? Il peut être intéressant de donner ici le sentiment de quelques historiens qui ont narré les faits. M. Joseph-Noël Fauteux écrit:

La même politique ostracisante demeura en vigueur jusqu'à la fin du régime français...

Un peu plus loin, à la suite d'autres prohibitions de même espèce, l'historien ajoute:

En effet, jusqu'à la fin, les habitants du Canada durent dependre de la Métropole pour les choses nécessaires à la vie. La colonie n'avait pas droit à son indépendance économique. Elle n'existait pas pour elle-même, mais uniquement en fonction du royaume de France. ${ }^{14}$

A l'inverse, M. Paul-Emile Renaud conclut:

L'incident n'est qu'une verrue inévitable et justifíe à la politique générale de liberté et d'assistance. ${ }^{15}$

Plus nuancé, E.-Z. Massicotte se contente d'observer:

Mais il n'entrait pas dans les vues des ministres européens de donner aux colons canadiens l'occasion de se détacher des travaux agricoles et de l'industrie domestique; peutêtre aussi leur répugnait-il de nuire aux favoris qui bénéficiaient du monopole que possédait sur le castor la compagnie des Indes". 16

12. AC, Coll. Moreau de St-Méry, F 3, 8: fol. 149.

13. Mémoire du roi, 15 mai $1736, \mathrm{AC}, \mathrm{B} 64^{3}: 597-625$.

14. Joseph-Noël Fauteux, op. cit., 2: 490, 492.

15. Paul-Emile Renaud, op. cit., 384.

16. "L'Anéantissement d'une industrie canadienne sous le régime français". BRH, 28 (1922): 200. 
De ces trois jugements, lequel s'approche le plus de la vérité? Pour y voir clair, il convient tout d'abord, dans l'histoire de l'économie coloniale, de distinguer nettement deux périodes: celle du temps de Colbert et celle d'après Colbert. En dépit de son système, le grand ministre appliqua avec un rare libéralisme le "pacte colonjal". Le "Mémoire du Roi pour servir d'instruction à Talon" (27 mars 1665) contient ce passage significatif:

Il observera que l'un des plus grands besoins du Canada est d'y establir des manufactures, et d'y attirer des artisans pour les choses qui sont nécessaires à l'usage de la vie; car jusques icy il a fallu porter en ce pays là des draps pour habiller les habitans, et mesme des souliers pour les chausser, soit qu'estant obligøs de cultiver la terre pour leurs subsist ${ }^{\text {ce }}$ et celle de leurs familles ils en ayent fait leur seule et leur plus importante occupation, soit par le peu de zèle et d'industrie de ceux qui les ont gouvernez jusqu'à présent. C'est pourquoy il examinera tous les moyens que l'on pourra embrasser pour l'introduction d'une chose si utile aud. pays, à laquelle Sa $\mathrm{Ma}^{\text {té }}$ contribuera par l'ouverture de ses coffres, estant bien persuadée qu'elle ne sçauroit employer une bonne somme d'argent à un meilleur usage. ${ }^{17}$

Qu'il s'agisse bien là d'une directive qui est autant de Colbert que du roi, on en trouve la preuve dans la lettre de Talon au ministre, 13 novembre 1666, où l'intendant dit expressément:

Quand j'ay demandé quelque secours pour Introduire ycy et y ayder plustost la fabrique que les manufactures, J'ay eu Intention de seconder les vostres, qui me paroissent dans l'article quinziesme de mon Instruction qui me dit en ces termes parlant des manufactures... ${ }^{18}$

Ce génie réaliste avait compris, comme Richelieu, du reste, l'élémentaire vérité qu'une colonie naissante fondée sur le défrichement agricole et par conséquent de lents et maigres revenus, devenait pure chimère, s'il lui fallait acheter ses biens de première nécessité. La guerre de Hollande et tout autant ses incidences trop négligées par les historiens, - "prodigieuses dépenses" pour Versailles, pour Marly, Clagny, Chambord, Saint-Germain, Fontainebleau, etc. ${ }^{19}$ - ne changent

17. RAPQ (1930-1931): 9 .

18. RAPQ $(1930-1931): 56$.

19. C.-J. Gignoux, Monsieur Colbert (Paris, 1941), 179-81, 192. 
point au fond le libéralisme économique de Colbert. S'il croit le trésor royal incapable désormais de financer l'industrie coloniale, il ne prétend point l'empêcher de naître. C'est encore lui qui écrivait à Talon, le 5 janvier 1666:

Le moyen d'y establir des manufactures consiste plustost dans leur industrie et leur travail [il s'agit des colons ] que dans les secours que le Roy y peut donner...

Et Colbert se disait certain:

...que quand le Canada sera rempli d'une grande quantite de beste a laine et a Corne, on pourra par le moyen de leurs dépouilles, \& de leur peau, manufacturer des Draps et autres estoffes, et des Cuirs que l'on convertira en divers usages a la commodité, et a l'avantage des habitans. ${ }^{20}$

Dans les années qui vont venir, Colbert ne cessera point de prêcher a Frontenac, par exemple, la nécessité d'encourager l'établissement de manufactures.

...il sera nécessaire que, suivant vos instructions, vous portiez toujours les habitans à s'adonner aux manufactures dont ils auront besoin pour leur usage, et en mesme temps a tout ce qui peut leur donner moyen de faire commerce par mer avec les Isles de l'Amérique et le Royaume...21

...A l'égard des pesches, des manufactures de potasse, goldron et autres, du commerce avec les Isles et avec ce Royaume, Sa Majesté veut que vous y donniez une application tout entière, et que vous y portiez les habitans par tous les moyens que vous estimerez convenables, parce qu'il n'y a rien qui produira tant d'advantages aux habitans ny qui contribuera plus à peupler le paĩs que ces establissements, et pour y parvenir elle veut que vous donniez une entière protection au nommé Follin qui travaille à l'establissement de la potasse, à ceux qui travaillent au goldron, et généralement à tous ceux qu'elle y envoyera ou qui iront d'eux-mêmes pour y faire de nouveaux establissemens dans lesquels ils réussiront... ${ }^{22}$

...Elle veut de plus que vous excitiez les mesmes habitans à continuer la manufacture de potasse, n'y ayant rien qui soit plus important pour l'augmentation de cette colonie que de chercher des marchandises et establir des manufactures

20. RAPQ (1930-1931): 43.

21. RAPQ (1926-1927): 10 .

22. RAPQ (1926-1927): 59 . 
et des pesches par le moyen desquelles les marchands puissent avoir du commerce soit avec le Royaume soit avec les isles de l'Amérique...23

...Il ne me reste qu'à vous rétérer les ordres que je vous ay donné toutes les années préédentes d'exciter toujours les habitants au commerce maritime, à l'establissement des manufactures et à la pesche, estant certain que ces trois points sont des moyens très faciles de produire l'abondance dans le pays, et par consequent la multiplication des habitans... ${ }^{24}$

Duchesneau reçut les mêmes consignes. Son mémoire à Colbert du 13 novembre 1680 en fait foi:

Le Roy et vous Monseigneur me commandez d'exciter tous les habitans aux manufactures et commerce par terre et par mer aux pesches ordinaires et sedentaires et a faire valloir les mines. ${ }^{25}$

Avec le temps, et avec ceux que M. C.-J. Gignoux appelle les "héritiers ingrats" de Colbert, le libéralisme métropolitain aura tendance à diminuer singulièrement. En 1683, l'année même de la mort de Colbert, l'ère débute, dans les finances de l'Etat, des déficits chroniques, déficits qui vont s'accumuler au rythme accéléré. Les colonies auront donc plus que jamais à se débrouiller seules. Pourtant les instructions à Callières qui sont du 27 mai 1699 - les colonies relèvent alors de Louis Phélipeaux de Pontchartrain - contiennent encore quelque chose de l'ancienne politique. Callières devra rendre compte des progrès de la culture, du lin et du chanvra. Les tuiliers, potiers et "briquetiers" envoyés au Canada ont-ils fait des établissements? De quelle manière subsistent-ils ? Où en sont les moulins à scie ? Y aurait-il possibilité de les multiplier ? ${ }^{26}$ L'autorité métropolitaine s'intéresse égalıment à l'école des Arts et métiers des Frères Hospitaliers de Montréal. Les Lettres patentes de l'institution (30 mai 1699) contiennent ce considérant: "Les arts, manufactures et mestiers estans des moyens propres et surs pour faire fleurir le commerce dans les Es-

23. RAPQ (1926-1927): 84.

24. RAPQ (1926-1927): 91 .

25. AC, C 11A, 5: 144-79.

26. AC, F 3, 8:207-27. 
tats". ${ }^{27}$ Mais voici venir la fin du grand règne. En 1703 la culture du chanvre et $d u$ lin vient de s'implanter avec succès au Canada. Il est question d'en faire des toiles; Vaudreuil et Beauharnois demandent qu'on envoie des tisserands. ${ }^{28}$ Le roi répond par un refus catégorique; et il en profite pour définir sa façon d'entendre désormais, au chapitre des manufactures, le pacte colonial:

...son intention n'a jamais esté qu'on fist des toiles en Canada pour pouvoir se passer de celles de France ainsy elle ne lé fera point envoyer de Tisserands elle a seulement pretendu que les habitans envoyasse' ces Chanvres en France pour y estre vendus soit a $\mathrm{Sa} \mathrm{Ma}^{\text {té }}$. pour le service de ses Vaisseaux soit aux particuliers afin de se passer par ce moyen de ceux des Estrangers et en general ils doivent observer que tout ce qui pourroit faire concurrence avec les manufactures du Royaume ne doit jamais estre fait dans les colonies lesqu'elles au contraire on ne scauroit trop employer a fournir les matieres necessaires aux manufactures du Royaume de sorte qu'on puisse s'il se peut se passer des matieres des Estrangers ils doivent regarder cela comme un des objets principaux des colonies qu'on n'establit que pour l'utilité des pays qui les forment et jamais dans l'intention de se passer de ces pays. ${ }^{29}$

Le couplet pourra encore varier, mais très peu. Deux ans plus tard, le 9 juin 1706, le comte de Pontchartrain rappelle à Raudot qu'il "ne doit pas décourager l'établissement de manufactures [au Canada], mais ne doit pas "les encourager au détriment de celles de France". 30 Cependant en 1704 l'on avait envoyé à Vaudreuil et Beauharnois deux potiers de terre demandés par eux. ${ }^{31}$ D'autre part, en 1728, Maurepas se prononce contre l'établissement de poteries et de verreries, matières "trop fragiles et d'un trop petit objet" pour exportation en France. ${ }^{32}$ Variation semblable au sujet du chanvre. Après avoir défendu de l'ouvrer en toiles au Canada et ordonné de l'expédier brut en France, on recommande en 1732 de l'ouvrer dans la colonia, sinon pour le tout, au moins pour une part, en cordages et en fil goudronné. On accepte même de faire passer ici des techniciens en corderie. Le

27. AC, F 3, 8: 262-65.

28. RAPQ (1938-1939): 15 .

29. RAPQ (1938-1939): $31-32$.

30. RAPQ (1940-1941): 389 .

31. RAPQ (1938-1939): 37 .

32. Maurepas à l'intendant Dupuy, 11 mai 1728, AC, C 11A, 50: 359-72. 
Conseil de marine voyait là un moyen d'augmenter le commerce par la consommation des cordages au Canada et à l'Ile Royale. Et le fil goudronné, disait-on, tiendrait moins de place que le chanvre brut dans les vaisseaux. ${ }^{3} 3$ Jusqu'à la fin du régime, l'autorité métropolitaine se renfermera dans la même méfiance à l'égard də certaines formes de l'industrie coloniale. C'est ainsi qu'en 1748 le ministre Maurepas s'inquiète soudainement des proportions prises au Canada par l'industrie des étoffes de laine. La guerre entre la France et l'Angleterre force les colons à l'élevage du mouton et à la remise en train des métiers. Il ne faut pas nuire au "débouchement" des manufactures de France, rappelle le ministre. Et il ajoute qu'il ne sera pas "difficile de procurer aux habitants le débouché de leurs laines dans quelques quantités qu'elles puissent être et il y aurait de l'inconvenient à les leur laisser consommer". 34

Ces interventions permettent-elles de conclure à un rigide protectionnisme, hostile à la moindre initiative coloniale dans le domaine de l'industrie? Et le pouvoir métropolitain se serait-il montré plus sévère au Canada qu'aux Antilles? On sait que, dans les Iles, par exemple, l'établissement de raffineries de sucre y fut interdit, au profit des raffineries de France. Le monopole du tabac, réservé à un fermier de l'Etat qui jouait avec les prix, entraînait, à Saint-Domingue, une surproduction ruineuse pour les cultivateurs. ${ }^{35} \mathrm{Au}$ Canada, en revanche, que d'espèces d'industries l'au torité métropolitaine toléra et même encouragea, si peu que ce fat. Citons la construction navale, la fabrication du fer, du goudron, des cordages, du brai, de la résine, de la potasse, de la térébenthine, de la chaux, de la tuile, de la brique, de la colle de poisson, de la bière, du cidre. Au vrai l'autorité ne fit grise mine qu'aux industries qui pouvaient empêcher, comme l'on disait, le "débouchement" des industries de France.

La distinction faite, entre deux époques: celle du temps de Colbert et celle d'après Colbert, une autre s'impose entre l'attitude de la me-

33. Conseil de marine a Hocquart, 22 avril 1732, AC, B 57-1: 221-22.

34. AC, B 87: 212.

35. Yvonne Bézard, Fonctionnaires maritimes et coloniaux sous Louis XIV Les Begon (Paris, 1932), 40, 49, 102. 
tropole et celle des autorités coloniales. Constamment, gouverneurs et intendants, c'est un hommage à leur rendre, se portent à la défense des entreprises des colons. Vivant dans le pays, ces hauts fonctionnaires en connaissent les besoins et les misères; ils savent qu'il suffit d'une année de disette dans les champs, d'une menace de guerre, du naufrage d'un vaisseau du roi, pour réduire à néant le pouvoir d'achat des habitants, raréfier à tel point les articles de consommation, dans les magasins du roi, des négociants, ou dans la pacotille des forains, qu'il n'y ait plus ni de quoi manger ni de quoi se vêtir. Il arrive même que la disette paralyse tous les travaux, sans excepter ceux du roi. Comme Talon, comme Denonville, gouverneurs et intendants savent encore que les fils de colon n'ont pas tous la vocation paysanne, et que, pour les préserver de l'oisiveté, de la course des bois, il faut leur ouvrir des carrières. Tous seraient donc d'avis de laisser se développer assez largement l'industrie coloniale. On l'a vu plus haut: maintes fois le ministre ou le roi se verront contraints de leur servir des rappels à l'ordre. En tête de ces hauts fonctionnaires, par ordre chronologique et par ordre de mérite, plaçons, après Talon, l'intendant Jacques de Meulles (16821686). De Meulles arrive ici du vivant de Colbert et cela peut expliquer bien des choses. D'un coup d'œil, l'intendant a discerné la situation économique des colons; elle pourrait se définir: la pauvreté dans l'abondance. Chacun a de quoi assez largıment: blé, légumes, viandes, mais sans débit possible, soit par absence de commerce à l'étranger, soit par l'impuissance d'achat du marché intérieur, dans une colonie trop peu peuplée et où d'ailleurs la possession des mêmes denrées par chacun empêche l'échange. Donc, pas d'argent dans la poche de l'habitant pour se procurer ses nécessités; et, par surcroît, obligation d'acheter ces nécessités, en particulier le vêtement, aux magasins du roi, deux fois plus cher qu'en France. Ce qui manque encore dans la colonie, ce n'est ni la laine, ni le cuir, ni la matière à fabriquer des chapeaux; ni le chanvre qu'on pourrait facilement faire pousser; ca qui manque, ce sont des manufactures et des ouvriers experts et à bon marché. De Meulles prône donc, et d'urgente nécessité, l'établissement de manufactures. Il y a, de lui, tout un plan de fabrique assez ingénieusement combiné avec une école d'arts et métjers. La fabrique, il proposait de l'établir dans l'ancienne brasserie de Talon. Le roi aurait envoyé, pour former des apprentis, deux ouvriers de quatre sortes de métiers: cordonniers, chapeliers, tisserands, sergers. Pour 
les débuts l'on aurait importé du cuir de Poitou et du chanvre de Rochefort. Il en aurait coaté à Sa Majesté, pour toute cette mise en train, $3,000 \#$, mais à remboursement facile par la vente aux colons, et au même prix qu'en France, des produits manufacturés. ${ }^{36}$

L'intendant Jean-Bochart de Champigny, successeur de de Meulles et qui vécut ici une période de guerre incessante (1686-1702), n'eut guère le temps de s'adonner aux œuvres de paix. On relève pourtant ce passage de l'une de ses lettres au ministre, à propos de la culture du chanvre et du lin et de la nécessité de manufactures au Canada:

L'habitant serait heureux s'il n'estoit pas obligé d'achepter des Estoffes et des thoilles de france qui coutent icy le double, ce qui les oblige d'employer tout ce qu'ils ont à s'en fournir. ${ }^{37}$

L'homme, toutefois qui, au temps de Champigny, a vu le plus clair dans la situation économique des colons, c'est le marquis de Denonville, ce gouverneur si méconnu. Denonville qui possédait assez peu, à ce qu'il semble, l'étoffe d'un homme de guerre, avait peut-être celle d'un grand administrateur. Il fut, en tout cas, l'un de nos rares gouvernants qui eut, ce que l'on pourrait appeler une politique de la jeunesse. Un fait l'a frappé: le désœuvrement de la jeunesse canadienne. Ce n'est pas lieu de dire ici ce qu'il avait rêvé pour la jeunesse des manoirs. L'autre jeunasse, celle des côtes, femmes et filles, lui parut aussi désœuvrée. Tout ce monde-là vivait dans la pauvreté, par la faute d'une production trop saisonnière et par manque d'industries vestimentaires. Plus de métiers à tisser en activité, disait Denonville, en dépit du soin que s'est donné pour cela M. Talon, si ce n'est dans la côte de Beaupré, "mais ce n'est que chez quelques particuliers". Et ce, observait le gouverneur, "dans le pays du monde le plus propre a faire des chanvres et du fil". Denonville proposait donc qu'on stimulât la culture du chanvre, l'élevage du mouton et qu'on fit venir de France des tisserands. Il recommandait fort l'école des métiers de Mgr de Lavai, "moyen admirable pour commencer un establissement de manufactures, qui sont absolument nécessaires pour le secours de

36. De Meulles au ministre, 12 nov. 1682, AC, C 11A, 6-1: 111-31; De Meulles au ministre, 4 nov. 1683, AC, C 11A, 6-1:282-329; Mémoire (non signé, sans date ni lieu, mais œuvre de de Meulles) AC, C 11A, 5: 121-23; Mémoire touchant le Canada et l'Acadie, AC, F 3, 2-1:237-78.

37. Champigny au ministre, 16 nov. 1686, AC, C 11A, 7: 320-49. 
ce pays". Il eât voulu qu'on dotât l'Evêque d'une "grosse abaye" pour lui permettre de développer son école. En même temps il eât souhaité commencer l'exploitation des forges de Saint-Maurice, et ne pouvait croire à l'impossibilité de fonder, un de ces jours, une verrerie. ${ }^{38}$ Toujours dans le même esprit, nous avons vu plus haut ce qu'avaient tenté, à leur tour, les Raudot, en faveur des industries du vêtement. ${ }^{39}$ L'intendant Michel Bégon na se montra pas moins zélé. ${ }^{40}$ L'intendant Dupuy, à qui il arriva d'avoir de bonnes idées, et même de fort bonnes, eût été favorable à qualques formes d'industrie locale: an particulier à l'industrie de la "peauserie", comme il disait, pour travailler les peaux qui ne pourraient pas se vendre, peaux d'ours, de bœuf illinois et surtout peaux de chevreuil très recherchées par les Anglais, ce qui fournissait prétexte à un commerce clandestin de ce côté. ${ }^{41}$ Gouverneurs et intendants resteront en ces dispositions jusqu'à la fin, tant qu'ils ne seront pas las d'être constamment rebutés par les gans de Versailles, et de n'avoir toujours à offrir à l'initiative des colons qu'une aide dérisoire: des encouragements en forme de belles paroles. Alors ils se replieront sur les industries complémentaires de l'économie métropolitaine, en particuljer sur la construction navale et l'exploitation des mines de fer. Pourtant en 1747, La Galissonnière et Hocquart, qui requjèrent une pension pour Mademoiselle de Repentigny, fille de l'entreprenante Madame de Repentigny, ne peuvent se tenir d'observer au ministre: "C'est un encouragement pour l'industrie, laquelle est plus nécessaire dans cette colonie que dans aucune au tre". ${ }^{42}$

Cette opposition entre autorités d'ici et de là-bas, l'affaire des chapeliers va tout particulièrement l'illustrer. Beauharnois et Hoc-

38. Mémoire de Denonville, 13 nov. 1685, AC, C 11A, 7: 48-87; Denonville au ministre, 8 mai 1686, AC, C 11A, 8: 2-43; Denonville au ministre, 10 nov. 1686, AC, C 11A, 8: 220-66.

39. Voir encore "Deux intendants au Canada sous Louis XIV", dans Bulletin de la Societé des sciences historiques et naturelles de l'Yonne (Auxerre) 7e vol.: 498.

40. Jean-Noël Fauteux, op. cit., 475.

41. Dupuy au ministre, 21 oct. 1726, AC, C 11A, 48: 260-79. - Le même au même et à la même date, AC, C 11A, 48: 253-59.

42. AC, C 11A, 87-1: 313. 
quart tentent presque l'impossible pour sauver la petite industrie de Coton et La Groy. Ils la défendent pied à pied. Le ministre leur a ordonné d'interdire toute fabrication de chapzaux de quelque espèce que ce soit, d'en empêcher la sortie de la colonie, d'avoir à détruire les établis. Ils répondent par une ordonnance qui enjoint tout au plus aux chapeliers de ne faire sortir de la colonie aucun de leurs chapeaux, sous peine d'encourir les châtiments ordinaires contre la fraude du castor. En suite de quoi ils prient le ministre de reconsidérer sa décision et d'approuver leur ordonnance. ${ }^{43}$ C'est alors - c'est-à-dire l'année suivante - que se produit l'intervention du roi: cassation de l'ordonnance du gouverneur et de l'intendant, ordre réitéré et sévère de procéder, sans retard, à la destruction de l'industrie. ${ }^{44}$ Beauharnois et Hocquart obéissent, mais ils ne laissent pas de solliciter une indemnité pour les malheureux chapeliers. La Compagnie des Indes opposa un premier refus. Beauharnois et Hocquart reviennent à la charge et gagnent le ministre lui-même à solliciter cette indemnité. ${ }^{45}$

Mais, au fait, dans l'ordre royal contre les chapeliers, faut-il voir une insigne illustration de la politique métropolitaine à l'égard de l'industrie coloniale? Les historiens auraient-ils eu raison d'interpréter l'ordre en ce sens? Pour parler plus nettement, l'arrêt plus ou moins extraordinaire visait-il à protéger, au premier chef, l'industrie du royaume et non d'autres profiteurs? Serait-il si difficile d'apercevoir, par exemple, dans la coulissa, et s'agitant plus que de raison, l'ombre des marchands de castor? Certes, gardons-nous de minimiser l'influence des chapeliers de la métropole. Il n'est pas permis d'ignorer qu'ən France, comme disait Riverin, le castor n'est propre à tout prendre qu'à faire des chapeaux. ${ }^{4} 6$ Mais cette industrie a pris, dans le royaume, des proportions assez considérables. Elle fabrique des coiffures pour "l'Europe, l'Amérique et autres païs". Elle vise à concurrencer avantageusement les chapeaux de fabrique anglaise. Et c'est à elle qu'à l'époque la Compagnie des Indes verse sa recette de castor. ${ }^{4} 7$ Une industrie coloniale de chapellerie qui aurait fait de l'exportation pouvait donc alarmer ces chapeliers. Et nous savons aussi qu'ils sur-

43. AC, B 62: 113-16.

44. Mémoire du Roi, 15 mai 1736, AC, B 64-3: 597-625.

45. Ministre à Beauharnois et Hocquart, 30 avril 1737, AC, B 65: 623; Ministre à M. de Fulvy, 27 janv. 1738, AC, B 67: 13-14.

46. Mémoire fait à Paris, 12 fév. 1705, AC, C 11A, 22.

47. Mémoire du roi, 15 mai 1736, AC, B 64-3: 597-625. 
veillent leurs affaires de près. En 1715 , alors qu'à la suitə de la dépréciation de la monnaie de carte au Canada et du retard dans le paiement des lettres de change, ils souffrent, depuis deux ans, de la disette $\mathrm{du}$ castor, ils proposent tout bonnement de se substituer au fermier de la traite. ${ }^{48}$ Ici-même au Canada, les négociants n'ignorent point la sorte de monopole que ces chapeliers peuvent au besoin constituer de la riche fourrure. Le 16 mai 1720 le roi déclare libre encore une fois le commerce du castor. La Compagnie des Indes se démet de son privilège contre un droit d'entrée sur cette fourrure dans les ports du royaume. Mais l'arrêt royal prohibe, en même temps, toute sortie dudit castor hors de France. Aussitôt les négociants du Canada de protester. Une telle prohibition, soutiennent-ils, susciterait un cartel des chapeliers du royaume qui, faute de concurrents, aviliraient le prix de la marchandise coloniale. ${ }^{49}$ Ajoutons qu'à cette coalition de chapeliers pouvaient se joindre parfois celle des manufacturiers du royaume, ceux d'Amiens, de Reims, de Rouen, de Beauvais, de Bordeaux, de Montauban, qui fabriquaient les marchandises d'échange pour la traite, étoffes de laine, draperies, merceries, toiles peintes, couvertures, et autres effets. Tous avaient intérêt au maintien de cette traite à bon niveau. ${ }^{50} \mathrm{Et}$ alors nous reposons la question: le coup est-il parti du milieu des manufacturiers? Rappelons que le niveau de la traite a bien de la peine à se maintenir vers 1735 . Autre fait par trop négligé, səmble-t-il, par les historiens qui ont épilogué autour de l'aventure des chapeliers canadiens. Qui paut oublier les fluctuations et les aléas de la traite du castor sous l'ancien régime ? Régime de liberté et régime du monopole, succession réitérée de celui-ci à celui-là; essai de l'affermage, essai de la régie, formation de compagnies après compagnies, dont la plupart trouvent à grand'peine leur assiette: autant d'avatars qui démontrent la précarité de ce commerce. Hier c'était la guerre iroquoise combinée souvent avec la guerre anglaise qui constituaient le pire achoppement. Depuis la paix d'Utrecht, deux fléaux non moindres ont encore tout compromis: la fraude du castor et la guerre froide qui sévit en Amérique du Nord, je veux dire ce jeu de souque à la corde où l'on voit, malgré la paix et à la faveur même de la paix bốteuse de 1713, Anglais et Français tâchant à étendre leur frontières,

48. AC, C 11A, 35: 387-88.

49. Vaudreuil et Bégon au ministre, 6 nov. 1720, AC, C 11A, 42: 114-21.

50. AC, C 11A, 50: 348-49; AC, C 11A, 60: 55-56. 
chacun de son côté, et chacun aussi s'efforçant d'attirer dans son rayon les chasseurs de fourrures. La fraude ou le coulage vers les colonies anglaises, comme c'est chose facile en un pays où le castor, resté monnaie, passe de main en main avant d'aboutir aux magasins de la Compagnie, et où on peut le porter librement soit au bureau de Montréal, soit a celui de Québec. ${ }^{51}$ Point d'années où le roi, le ministre ne profèrent des plaintes, n'exhortent gouverneurs et intendants à sévir contre les contrebandiers et prennent parfois contre les chefs de la colonie le ton comminatoire. Longtemps les arrêts les plus sévères n'y pourront rien ou très peu. Comment réprimer, en effet, cette contrabande dans un pays ouvert et immense d'où la fourrure peut s'échapper par tant de voies: par la frontière du sud, par cella de l'ouest, par le fleuve, à bord des vaisseaux marchands en partance de Québec et même par les vaisseaux de pêche du Labrador, vaisseaux de Saint-Malo, de Bayonne et de Marseille. ${ }^{52} \mathrm{~A}$ la fraude chacun semble se livrer. D'après l'intendant Dupuy, tout Montréal est infesté de contrebande dans tous les ordres et les Etats, sans en excepter aucun; soldats et commandants de la garnison sont les premiers a la favoriser. Chacun y a ses magasins; on passe la marchandise sous la robe et la jaquette. ${ }^{5} \mathrm{La}_{\text {fraude, elle }}$ se fait par l'intermédiaire des sauvages domiciliés, ceux du Saut SaintLouis, ceux du Lac des Deux-Montagnes, qui ont liberté d'aller où ils veulent; ${ }^{54}$ elle se fait par les Anglais des colonies du sud qui, sous toutes sortes de prétextes: apprendre le français, recouvrer des dettes, font un voyage ou un petit séjour à Montréal. ${ }^{55}$ Elle se fait par les négociants de la même ville qui, depuis longtemps, se servent des sauvages domiciliés pour passer leur castor à l'étranger; ruinés par l'incendie de $1734,{ }^{56}$ ces négociants, pour se refaire, sont pris comme d'une fringale de fraude. Et, si nous en croyons Hocquart, rien de plus illusoire, que d'espérer quelque dénonciation entre tous ces gens liés par le sang et l'intérêt. ${ }^{57}$

51. AC, C 11A, 48: $64-72$.

52. Hocquart aux directeurs de la Compagnie des Indes, AC, C 11A, 51: 28795. - AC, C 11A, 64: 63-74.

53. Dupuy au ministre, 20 oct. 1727, AC, C 11A, 49: 353; ibid., 49-2: 355-64.

54. AC, C 11A, 67: 63-103; AC, C 11A, 69: 75.

55. Hocquart au ministre, 25 oct. 1729, AC, C 11A, 51: 249-50.

56. AC, C 11A, 61: 110-11; AC, B 49-2: 380-414; AC, C 11A, 67: 63-103.

57. Hocquart aux directeurs de la Compagnie des Indes, 10 et 20 oct. 1729, AC, C 11A, 51: 287-95. 
Le coulage du castor vers le sud, c'est encore, à la même époque que les Anglais vont lui donner un effroyable accroissement par leur coup de maître de 1727 à Choueguen. L'établissement du fort de ce nom, au sud du lac Ontario, presque en face du fort Frontenac, qu'étaitce sinon le coin enfoncé au cœur même de l'empire du castor, una bifurcation extrêmement périlleuse introduite sur la route des fourrures par les lacs? En France et à Québec on a ressenti durement le coup. Pour déloger l'assaillant, on parut même résolu à l'emploi de la force. ${ }^{58}$ Puis on subit ce qu'on n'osait empêcher. Et le coulage du castor par Choueguen prit le caractère d'un torrent. Ni le renforcement du fort Niagara, ni les défenses faites aux voyageurs et aux Indiens des lacs, de descendre par le sud du lac Ontario ne purent fermer aux flottilles la route séductrice. Les sauvages les plus éloignés s'en allèrent porter leurs pelleteries a Choueguen. ${ }^{59}$ Les Anglais y payaient le castor plus cher que la mesquine Compagnie des Indes; ils offraient une marchandise de troc de meilleure qualité et d'un prix inférieur à celui de la même Compagnie; surtout l'eau-de-vie y coulait a flot. Pour comble, un mandement de Mgr Dosquet du 26 novembre 1730, mettait fin au régime de tolérance d'alors sur la vente de l'eau-de-vie aux sauvages. L'évêque faisait un cas réservé à lui seul de "ceux qui directement ou indirectement contribuent à enivrer les sauvages". 60 Par la suite, et sur les instances des autorités, l'évêque aura beau apporter des tempéraments à sa décision. ${ }^{61}$ Le mandement va causer, dans les Pays d'en haut, une extraordinaire perturbation. Effrayés par le cas rêservé, les gardes-magasins se refusent à toute vente d'alcool. Dépités, les sauvages se mettent à passer tout droit à Niagara et reviennent de Choueguen, délestés de leur castor, mais chargés de marchandises anglaises et surtout d'eau-de-vie qu'ils détaillent dans leurs villages. ${ }^{62}$

Faisons maintenant la conjonction de tous ces faits ou circonstances: fraude ou contrebande, attirance de Choueguen, cas réservé de l'évêque, guerre des Renards et dérangements dans les Pays d'en haut, mesquineries de la Compagnie des Indes; et l'on aperçoit quels contrecoups en va recevoir la recette du castor. Avant 1736, cette recette a

58. AC, B 48-2: $135-39$.

59. AC, C 11A, 67: 40-62.

60. Mandements, lettres pastorales et circulaires des Evêques de Québec, publiés par Mgr H. Têtu et l'abbé C.-O. Gagnon (6 vol., Québec, 1887-90), 1: 535-37.

61. Beauharnois et Hocquart, 10 oct. 1735 , AC, C 11A, 63: 44-84.

62. Ibid. 
considérablement augmenté. De 170 milliers en 1732, elle est passée à 221 milliers en $1733 ;^{63}$ en 1734 , le fléchissement s'affirme désastreux, imputable selon Beauharnois et Hocquart, aux mouvements de guerre dans les Pays d'en haut, mouvements qui ont empêché les sauvages de chasser, mais imputable aussi à la concurrence de Choueguen et à l'eau-de-vie qui s'y vend. ${ }^{64}$ Une légère remontée s'opère en 1735: 78 milliers environ font la recette, mais remontée attribuable, pour plus de la moitié, à la découverte de l'Ouest et de cette nouvelle source de castor. ${ }^{65}$ Un nouveau fléchissement ne tarde point qui, en 1737, ramène la recette à 124 milliers. ${ }^{6}$ Puis, voici quatre ans qu'aux forts Niagara et Frontenac, les revenus de la traite qui étaient auparavant de 52,000\# par année, sont tombés à 25 ou 35,000\#. Désormais, selon Hocquart et Beauharnois, les Anglais, par Choueguen et avec laur eau-de-vie, tirent plus de castor du Canada que les Français. ${ }^{67}$

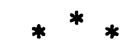

C'est en toutes c ss incidences que, pour les bien entendre, croyonsnous, il faut replacer l'intervention du roi contre les chapeliers canadiens. On se défend mal de panser à un peu d'affolement. Ces messieurs de la Compagnie des Indes auraient cru, semble-t-il, le commerce du castor en train de crouler tout de bon. Car ne serait-ce pas ces messieurs, plus encore que les industriels et plus même que les chapeliers de France, qui auraient emporté la décision royale? Relisons ce document. Les motifs qui paraissent l'avoir provoqué, éclairent passablement toute l'affaire. On n'y trouve point le couplet ordinaire sur la nécessité d'une subordination de l'industrie coloniale à la métropolitaine. En revanche, on y relève deux considéran ts qui, s'ils ne s'expliquent par un peu d'affolement, deviennent d'une parfaite puérilité. Le roi-ou plu tôt ceux qui l'inspirent - font gravement observer que l'introduction en France des chapeaux demi-foulés de Québec signifierait l'anéan-

63. Hocquart au ministre, 25 oct. 1732, AC, C 11A, 58: 67-69; Hocquart au même, 24 oct. 1733 , AC, C 11A, 60: 104-105.

64. Hocquart au ministre, ler nov. 1734, AC, C 11A, 62: 55; le ministre a M. de Fulvy, 27 déc. 1735, AC, B 62: 113-116.

65. Hocquart au ministre, 25 oct. 1735 , AC, C 11A, 64: 75-81. 67: 186 . 195 .

66. Hocquart aux directeurs de la Compagnie des Indes, oct. 1737, AC, C 11A,

67. Beauharnois et Hocquart au ministre, 12 oct. 1736, AC, C 11A, 65: 17-44; mémoire non signé, apparemment de Hocquart, AC, C 11A, 67: 40-62. 
tissement du monopole de la Compagnie des Indes, et aussi l'anéantissement de la fabrique des chapeaux du royaume. ${ }^{68} \mathrm{Ne}$ sont-ce pas là de bien grands mots a propos d'une entreprise que Beauharnois et Hocquart désignent comme "une si petite fabrique", qu'elle "ne peut faire un objet considérable, jetant à peine par an, sur le marché environ 12 à 1,500 chapeaux". D'autant que ces chapeaux de fabrication canadienne devaient passer en France pour y être "perfectionnés", ce qui veut dire pour tomber entre les mains des chapeliers de là-bas. Au reste, ces considérants ne rimaient plus à rien, après l'ordonnance du gouverneur et de l'intendant qui interdisait l'exportation des chapeaux demifoulés. ${ }^{6}$ De même le danger était-il appréciable, pour la chapellerie métropolitaine, d'une concurrence sur le marché colonial ? Si l'on tient compte que, vers 1705 , un chapeau de castor se vendait de 40 à 50 écus, ${ }^{70}$ on a peine à croire que la coiffure se put trouver sur toutes les têtes. Notons encore qu'en 1736, lorsque le roi revient à la charge et sévit tout de bon, il se dérobe assez prestement au sujet de l'ordonnance de Beauharnois et de Hocquart; il n'en dit mot; en de solennelles considérations il préfère se rabattre sur le rôle et l'usage du castor, commerce qui "intéresse l'Etat", qui en "occasionne un réciproque entre la france et le Canada". ${ }^{71}$

Il semble que la lumière commence à se faire. Mais poursuivons. Il a été dit plus haut de quel camp venait l'alerte au ministre. Qui maintenant paraît bien avoir dicté la décision finale ? Elle apparaît dans un mémoire du roi. Ce mémoire du roi est-il bien du roi ? Comme chacun sait, il y a déjà longtemps qu'à Versailles, et depuis la mort de Louis XIV, les affaires coloniales sont plus ou moins abandonnés à des fonctionnaires subalternes, anciens gouverneurs, ancien intendants des colonies, tel par exemple, Raudot après son retour en France. Ce sont ces fonctionnaires ou d'autres de plus modeste rang, qui souvent cuisinent mémoires et dépêches. ${ }^{72}$ Presque en toutes choses, d'ailleurs, le ministre est tout-puissant. Et bien avisé qui pourrait y découvrir la part du souverain. M. Pierre Gaxotte, dans son Siècle de Louis XV

68. Le roi a Beauharnois et Hocquart, 4 avril 1735, AC, C 11A, 63: 30-58.

69. Beauharnois et Hocquart au ministre, 10 oct. 1735, AC, C 11A, 63: 44-84.

70. AC, C 11A, 36: 425 .

71. Mémoire du roi, 15 mai 1736, AC, B 64-3: 597-625.

72. Voir Albert Duchesne, La Politique coloniale de la France (Paris, 1926), 56. - Francis H. Hammang, The Marquis de Vaudreuil - New France at the beginning of the eighteenth century - Part 1: New France and the English colonies (Bibliothèque de l'Université, Louvain, 1938), 21-26. 
(p. 378), s'est amusé à marquer la différence entre les deux locutions: le Roi en son Conseil et le Roi étant en son Conseil, la première s'appliquant aux arrêts où Sa Majesté n'est présente que par son fauteuil et son chancelier; la seconde, aux arrêts rendus en sa présence effective et personnelle. Mais encore une fois, en cette affaire de chapeaux et de castor, de qui a pu dépendre le dernier mot? Ebranlé par les observations de Beauharnois et de Hocquart, le ministre était prêt à une solution de compromis. Le 27 décembre 1735, il écrivait, en effet, à un M. de Fulvy, pour lui faire part des observations reçues de Québec et surtout pour lui marquer l'insignifiance de la chapellerie canadienne:

Il paroit en effet que la Compagnie des Indes n'en peut recevoir aucun dommage, et qu'au moyen de l'Ordonnance qu'ils [ Beauharnois et Hocquart ] ont rendue, les manufactures de france n'en souffriront pas non plus. ${ }^{73}$

Qui donc, à ce moment, devint le suprême arbitre? Le ministre ajoutait, dans cette même lettre à ce $M$. de Fulvy:

Je n'ay cependant point encore proposé au Roy d'approuver cette ordonnance; Et j'attendray, pour prendre les ordres de S.M. que vous ayés pris la peine de me marquer ce que Vous en pensés. C'est ce que je vous prie de faire; je vous envoye a cet effet Copie de cette même Ordonnance.

Je n'ai pu retracer la réponse de ce M. de Fulvy au ministre. Mais le coup - en pouvons-nous douter? - partit de ce puissant personnage. Ce M. de Fulvy (Jean-Louis Orry de Fulvy), conseiller d'Etat et intendant des finances, frère du célèbre Orry, contrôleur général des finances sous le Cardinal Fleury, était bel et bien à l'époque, gouverneur de la Compagnie des Indes. De ce grand joueur, que l'on s'accorde à reconnaître pour "un aigle en affaires", l'on a pu dire que "jamais la Compagnie des Indes ne fut mieux gouvernée que par lui". ${ }^{74}$ Rappelons-nous l'affolement des marchands de castor vers 1735; pensons aussi à la mesquinerie bien connue de la fameuse Compagnie en Louisiane comme au Canada, à son égoïsme d'affairistes moins avides de progrès colonial que de dividendes. Et il sera permis de conclure que la sentence de mort portée contre la chapellerie canadienne procédait moins du protectionnisme industriel de France que de l'intervention du

73. Le ministre à M. de Fulvy, 25 déc. 1735, AC, C 11A, B 62: 113-116.

74. Michaud, Biographie universelle. 
monopole du castor. Ce qui veut dire que l'on pourra encore invoquer l'incident pour définir la politique de la métropole française à l'égard de l'industrie coloniale, à la condition toutefois de ne le faire qu'avec discrétion.

Lionel Groulx, ptra président de l'Institut

Un cours d'bistoire à écouter. - Le président de l'Institut d'Histoire de l'Amérique française a commencé, le 4 décembre dernier, au poste C.K.A.C., de 4 h. 30 à 4 h. 45 , un cours d'Histoire du Canada. Le cours se répartira sur un ensemble de 90 à 100 leçons. Il aura lieu tous les dimanches, au même poste et à la même heure. C'est, comme on peut le voir, une entreprise d'envergure, patronnée par la Société Saint-Jean-Baptiste de Montréal. Ceux qui s'intéressent à l'histoire sont invités à se mettre aux écoutes. 